\title{
Behavior Change Following Diagnosis with Acute/Early HIV Infection-A Move to Serosorting with Other HIV-Infected Individuals. The NIMH Multisite Acute HIV Infection Study: III
}

\author{
Wayne T. Steward · Robert H. Remien · Jenny A. Higgins $\cdot$ Robert Dubrow \\ Steven D. Pinkerton · Kathleen J. Sikkema · Hong-Ha M. Truong • \\ Mallory O. Johnson · Jennifer Hirsch $\cdot$ Ronald A. Brooks · Stephen F. Morin
}

Published online: 6 June 2009

(c) The Author(s) 2009. This article is published with open access at Springerlink.com

\begin{abstract}
Risk reductions behaviors are especially important during acute/early HIV infection, a period of high transmission risk. We examined how sexual behaviors changed following diagnosis of acute/early HIV infection. Twenty-eight individuals completed structured surveys and in-depth interviews shortly after learning of their infection and 2 months later. Quantitative analyses revealed significant changes after diagnosis, including reductions in total partners and decreases in the proportion of unprotected sex
\end{abstract}

W. T. Steward $(\bowtie) \cdot$ H.-H. M. Truong

M. O. Johnson · S. F. Morin

Center for AIDS Prevention Studies, University of California,

San Francisco, 50 Beale St., Suite 1300, San Francisco,

CA 94105, USA

e-mail: wayne.steward@ucsf.edu

R. H. Remien - J. Hirsch

HIV Center for Clinical and Behavioral Studies, New York State Psychiatric Institute and Columbia University, New York, NY, USA

\section{J. A. Higgins}

Center for Health and Wellbeing, Princeton University,

Princeton, NJ, USA

R. Dubrow

Center for Interdisciplinary Research on AIDS, Yale University, New Haven, CT, USA

\section{S. D. Pinkerton}

Center for AIDS Intervention Research, Medical College of Wisconsin, Milwaukee, WI, USA

K. J. Sikkema

Duke University, Durham, NC, USA

R. A. Brooks

Center for HIV Identification, Prevention and Treatment

Services, University of California, Los Angeles, CA, USA acts occurring with uninfected partners (serosorting). Qualitative findings indicated that these changes were motivated by concerns about infecting others. However, participants were less successful at increasing the frequency with which they used condoms. These results suggest that the initial diagnosis with HIV may constitute an important component of interventions to promote risk reduction during the acute/early stages of the disease.

Keywords Acute HIV - Serosorting - Behavior change · HIV prevention

\section{Introduction}

Acute and early HIV infection play a critical role in the spread of the epidemic. Viral loads, and, consequently, infectiousness, are at their highest during the period of acute HIV infection (AHI; the several weeks to about 2 months between acquisition of HIV and completion of seroconversion) (Pilcher et al. 2004). Furthermore, although acute HIV shedding is over about 10 weeks post-infection, elevated onward transmission likely extends through the period of early infection (the 6 months after seroconversion) due to ongoing high risk behaviors, associated sexually transmitted infections that increase transmission risk, and transmission amplification through high-risk sexual and drug-use networks. Transmissions during acute/early infection may account for as many as half of new infections. (For more detailed discussion and references, see Kerndt et al. 2009, the first paper of this series in this issue of the journal).

Because risk reduction is especially critical during these stages of HIV disease, it is important to understand people's readiness and ability to adopt and sustain rapid behavior change after diagnosis with acute/early HIV infection. On 
the one hand, this time period presents special challenges: people are coping with a new diagnosis, including the challenge of obtaining needed medical care. They also may be experiencing illnesses associated with seroconversion. And, they may still face many of the same circumstances and factors that contributed to the risk behaviors that led to infection. On the other hand, previous research suggests that people can reduce transmission risks after testing HIVpositive (Colfax et al. 2002; DiFranceisco et al. 2005; Gorbach et al. 2006; Weinhardt et al. 1999). Understanding the degree to which people adjust sexual behaviors after diagnosis with acute/early HIV in particular-and examining how exactly they accomplish this goal and their motivations for doing so- is valuable for developing AHI risk reduction interventions. Such programs will need to build upon behavior changes prompted by initial diagnosis and offer support for longer-term risk reduction efforts.

This is the third in a series of five papers (see also Atkinson et al. 2009; Kelly et al. 2009; Kerndt et al. 2009; Remien et al. 2009) that describe results from the National Institute of Mental Health Multisite Acute HIV Infection Study (see Kerndt et al. 2009, for the overall aims of the study). In the analyses presented here, we examine changes in sexual risk behaviors, seeking specifically to understand the degree to which such changes are a product of reductions in partners, reductions in sex acts, increases in condom use, and use of serosorting and seropositioning. The latter two approaches are informal risk reduction techniques. With serosorting, individuals choose partners thought to have a similar HIV serostatus (Suarez and Miller 2001; Suarez et al. 2001). Recent studies have documented its increasingly widespread adoption, particularly among men who have sex with men (MSM) (Grov et al. 2007; Mao et al. 2006; Osmond et al. 2007; Parsons et al. 2005; Truong et al. 2006). With seropositioning, HIV serodiscordant male couples choose sexual positions in which the likelihood of HIV transmission is believed to be lower (e.g., the HIV-positive partner will be the receptive, as opposed to insertive, partner during anal sex) (Parsons et al. 2005; Van de Ven et al. 2002). Informal risk-reduction techniques can in theory reduce the likelihood of HIV transmission, but require accurate health information and thorough disclosure to be successful. Inaccurate serostatus disclosures prior to serosorting have already been shown to lead to increased disease transmission events (Butler and Smith 2007).

\section{Methods}

Participants were 18 years of age or older, had sufficient English proficiency to complete the study measures, and had documented evidence of acute or early HIV infection (see Kerndt et al. 2009). Participants were asked to attend two study visits: the first slated for within 4 weeks of when the participant learned of his or her diagnosis (T1) and a second slated for 8 weeks later (T2). At each visit, participants completed an in-depth qualitative interview and a structured quantitative survey (see Remien et al. 2009).

In the structured surveys, participants provided detailed information on their recent sexual history. Specifically, during their $\mathrm{T} 1$ interview, they were asked to describe their sexual behaviors in the 8 weeks before diagnosis and, separately, their behaviors in the days or weeks that had elapsed between diagnosis and the day of the interview $(M=$ 5.6 weeks; $\mathrm{SD}=3.0$ weeks). At their $\mathrm{T} 2$ interview, they described their behaviors in the weeks that had elapsed between the $\mathrm{T} 1$ and $\mathrm{T} 2$ interviews $(M=9.9$ weeks; $\mathrm{SD}=$ 2.5 weeks). Interviewers used a visual schematic to help participants remember start and end dates for these recall periods. For each assessed time period, participants provided separate counts of the number of HIV-positive, HIV-negative, and HIV-serounknown (i.e., unknown to the participant) partners with whom they had had oral, vaginal, and/or anal sex. Then, for each partner type, they provided counts of the number of times they had engaged in oral, vaginal, and anal sex. (As applicable, the assessments separately captured insertive and receptive acts.) If vaginal or anal sex acts were reported, participants also provided counts of the number of times that condoms were used. During the in-depth qualitative interviews, participants were asked to focus on two narratives of importance to behavior change analyses: their social and sexual lives before becoming infected with HIV, and their social, sexual, and preventive behaviors following diagnosis.

\section{Analyses}

For quantitative analyses, we calculated the total number of partners (stratified by serostatus), and the total number of vaginal and anal sex acts (stratified both by condom use and partner serostatus). Oral sex was not included in the analyses. Because the recall periods differed in length, we standardized the counts by dividing the number of partners and risk acts reported for each participant by the number of weeks that had elapsed in a recall period for that participant. For example, an individual who reported eight risk acts in an eight-week period would have a standardized count of one act/week. We then used paired-samples t-tests to assess the significance in changes across recall periods in the mean number of partners and mean number of sex acts, and chi-square analyses to examine changes in the relative distribution of partners and sex acts across partner serostatus and condom use strata.

For qualitative analyses, we coded and then sorted data following procedures described elsewhere (Remien et al. 
2009). For the findings reported here, coding reports focused on sexual behaviors before and after diagnosis, participants' perceived responsibilities and sense of altruism in making decisions about sex, and use of informal risk reduction strategies.

\section{Results}

Findings presented in this paper include only the 28 participants who completed both their $\mathrm{T} 1$ and $\mathrm{T} 2$ interviews. Twenty-two of the participants were diagnosed with AHI and six were diagnosed with early HIV infection. All but one were male and all but two were MSM. There were no differences in the characteristics of this subsample and the total participant sample $(N=34$; see Kerndt et al. 2009, for complete demographic characteristics).

\section{Structured Survey Results}

\section{Number of Partners}

Figure 1 displays the significant decline over time in mean reported number of weekly partners (before diagnosis: $M=1.23, \mathrm{SD}=1.60$; between $\mathrm{T} 1$ and $\mathrm{T} 2$ interviews: $M=0.50, \mathrm{SD}=0.94 ; t(27)=2.98, P<.006)$. Before diagnosis, $15 \%$ of all partners were described as HIVpositive. This proportion increased to $68 \%$ between diagnosis and T1 interview $\left(\chi^{2}=16.5, P<.001\right)$ and to $49 \%$ between $\mathrm{T} 1$ and $\mathrm{T} 2$ interviews $\left(\chi^{2}=5.9, P=.052\right)$.

\section{Number of Sex Acts and Proportion with Condom Use}

As shown in Fig. 2, there was no significant change in the mean total number of anal and vaginal sex acts per week (before diagnosis: $M=2.04, \mathrm{SD}=4.27$; between diagnosis and T1 interview: $M=2.07, \mathrm{SD}=4.14$; between $\mathrm{T} 1$

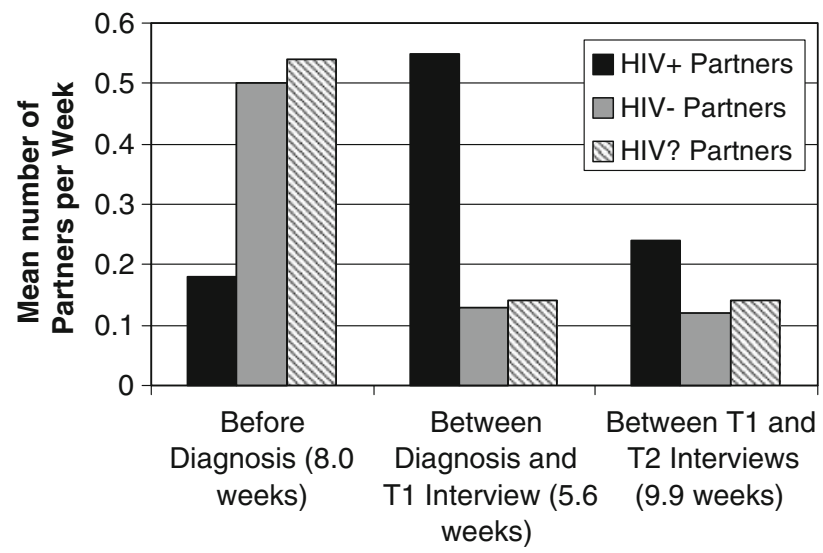

Fig. 1 Mean number of partners per week, stratified by partner serostatus

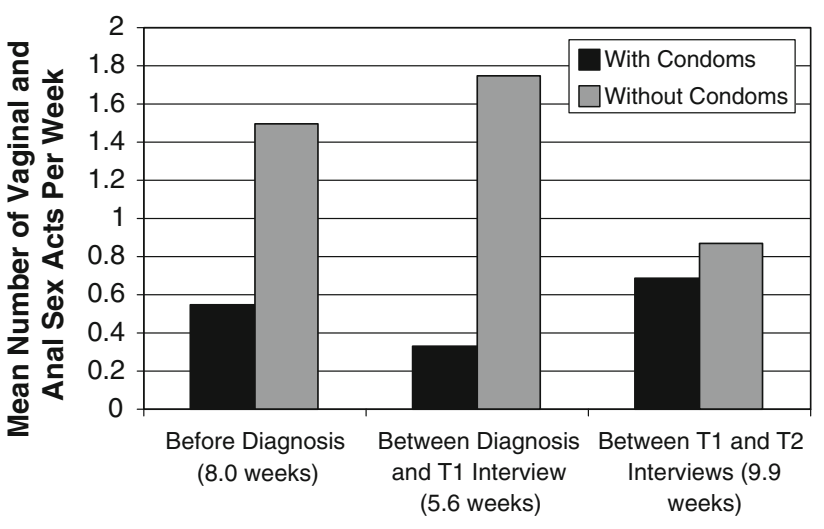

Fig. 2 Mean number of vaginal and anal sex acts per week, stratified by condom use

and $\mathrm{T} 2$ interviews: $M=1.55, \mathrm{SD}=1.69$ ). (The figure combines receptive and insertive anal sex acts among MSM, as there were no differences in the frequency of each during the three recall periods.) However, the proportion of condom-protected sex acts was marginally greater between $\mathrm{T} 1$ and T2 interviews (44\%) than before diagnosis (27\%) $\left(\chi^{2}=3.28, P=.07\right)$.

\section{Unprotected Sex and Partner Serostatus}

As shown in Fig. 3, 20\% of reported unprotected sex acts before diagnosis were with partners believed to be HIVpositive. This proportion increased markedly to $98 \%$ between diagnosis and T1 interview $\left(\chi^{2}=58.4, P<.001\right)$, and to $97 \%$ between the T1 and T2 interviews $\left(\chi^{2}=36.4\right.$, $P<.001)$. Given recent findings showing that inaccurate information about partners' serostatus contributes to transmission risks (Butler and Smith 2007), it was notable that only $21 \%$ of participants $(n=6)$ identified having engaged in any transmission risk behaviors with a known HIV-positive partner prior to diagnosis. For anal sex acts in male-male partnerships, it was also notable that there were no significant differences in the numbers of insertive and receptive anal sex acts occurring with HIV-positive,

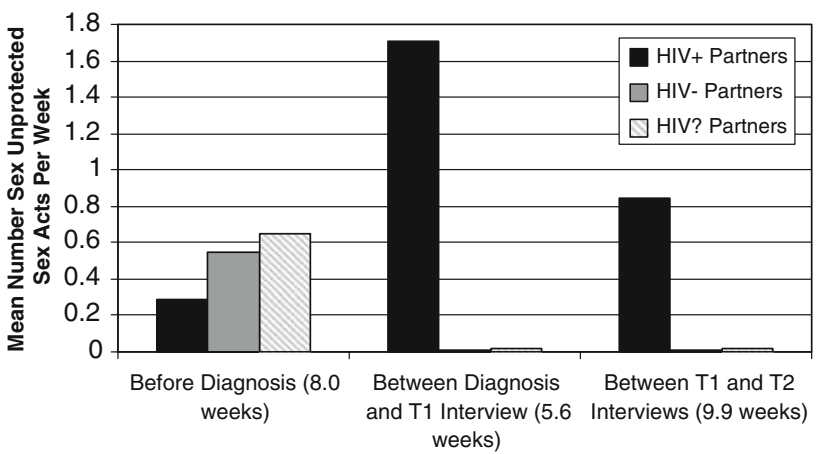

Fig. 3 Mean number of unprotected vaginal and anal sex acts per week, stratified by partner serostatus 
HIV-negative, and HIV-serounknown partners across the three recall periods.

In-Depth Interview Findings

In qualitative interviews, we sought to contextualize the patterns observed in the quantitative analyses and to understand participants' motivations for behavior change.

\section{Reducing the Total Number of Partners}

Engaging in sex with fewer partners was the most common strategy identified for reducing risk. For example, one participant who had five concurrent partners prior to diagnosis reported only one partner after diagnosis:

I've always had like more than one partner. [But]

I've just been more serious in our relationship than

before. So I'm just trying to... And my HIV status. I

don't want to infect other people. (20 year old gay

man, San Diego)

Another participant reduced his partners from two to one:

The two months before [diagnosis], I had sex with two people. [...] And now I am just with [my partner]. I think I am still afraid to get anything out of my relationship with him, and... I don't want to take a risk to hurt somebody else. I don't want to do it, I don't want to do it. So I would rather just not have it [sex]. (39 year old gay man, Providence)

\section{Temporarily Abstaining From Sexual Activity}

Some respondents described refraining from all sexual activity immediately after diagnosis. Their reasons for doing so were varied. Several were simply too ill or distressed:

I've been too sick. No interest. No interest. Not even masturbation. None of that. [...] You know, I haven't thought about sex, and I usually think about sex once a day. I felt insecure enough without having HIV. (57 year old gay man, San Francisco)

Fear of infecting others-or getting re-infected themselves_-prevented other respondents from having sex. For example, one participant went from an average of three to five partners a week to having none:

It's just lack of libido. That, and-and a concern, and like, you know...I could potentially kill someone. And I don't think I could handle that. So I don't. (26 year old gay man, New York)
Another participant expressed similar feelings when asked about his sexual future:

Well, I haven't had any sexual feelings. I don't-I'm sure probably later on I would, but now I haven't really been having any sexual thoughts or anything sexual enter my mind now. [...] You know, I know there's condoms but-like, in August, the condom popped. I don't want to have-or risk any-or put anybody at risk, and I think that's what really bothers me as far as having sex, you know? (22 year old gay man, New York)

\section{Increases in Safer Sex Practices}

Participants described both increases in condom use and attendant decreases in specific risk practices. For example, one man spoke of a previous habit of "poking around" partners' anuses with his penis, or having partners penetrate him before applying a condom:

Now, unsafe is really not an option. There's no more poking around the outside. We can't start sex without a condom and then put one on later. (35 year old gay man, San Diego)

By contrast, other participants described challenges in changing condom use behaviors:

Well, [my diagnosis has changed] two things. They're related but separate. One is my sex drive, and the other is the lack of enjoyment I get from sex I have. Now my sex drive is kind of bad. I wouldn't mind having sex three or four times a week, but I can't...I don't enjoy it very much. So I've been trying to find condoms that are more sensitive and I've experimented with brands and types and it doesn't seem to be helping, and it's frustrating. I don't know what it means for the long term. [...] I've never found condoms very satisfying. That was the primary reason that...that I started having lots of unprotected sex [before diagnosis]. (23 year old gay man, San Diego)

Seeking New (or existing) Partners who are HIV Positive

Diagnosis led a number of respondents to seek out partners who were HIV-positive. Most often, they found these partners through Internet profiles, introductions from friends, support groups, or other HIV social networks. Other participants returned to former partners whom they knew to be HIV-positive, or enhanced the intimacy of an existing relationship with an HIV-positive long-term partner. Primary reasons for their partner selection were concern about 
transmission risks and a belief that other infected individuals could relate to their experiences:

I don't want to be with someone who's negative, for two reasons. One, because they don't know what I've been through, and they'll never understand, and it's a part of me-again, maybe in a year from now, when it's not such a big deal [...], but not now. Also, I don't think that I could trust someone, or be $100 \%$ with someone, that I could be a threat to. (26 year old gay man, New York)

Another participant described a major reduction in partners as well as a new focus on partners who were HIVpositive:

Since I've been diagnosed, I've been back with my steady partner [who is HIV positive]. We're both pretty open about sex. We've played with two other people, both of them also being positive [...] It was unprotected. My general attitude I guess is I have the most potentially damaging disease out there. They're also positive. So I don't have to worry about giving them HIV. Most other stuff can be cured. (36 year old gay man, San Diego)

\section{Learning that Partners Were Already HIV-Positive}

Although many participants described concerted efforts to serosort after diagnosis, others came to realize all too late that existing partners already were positive:

After I got the notice that I was HIV positive, I went back and called everybody that I was playing with. And luckily I found out that half of them were positive [...] I called them and I said "You know what, this is what happened. So I hope you're doing okay. Go and check yourself." And then some came and said "Unfortunately I'm positive too." And, you know, I wasn't shocked but I had to take responsibility for what I had done also. So it was kind of upsetting [...] One of the guys came and said "Well I assumed you were-you knew that I was positive." And I said "Well how can I assume that you were positive? The last time we talked you told me you have Chlamydia. So if you had something else worse than Chlamydia I was expecting you to tell me." (37 year old gay men, San Francisco)

\section{Discussion}

Our findings suggest that diagnosis with acute/early HIV infection could play an important role in reducing transmission-risk behaviors, but also highlight the need for interventions to enhance and sustain these changes. After diagnosis with acute/early infection, participants in our study, nearly all of whom were MSM, reduced their number of partners and serosorted, limiting their unprotected intercourse to others they believed to be HIV-positive. (Seropositioning, another informal risk reduction strategy, did not appear to be used by our participants.) Although risk reduction efforts have also been observed among people diagnosed at later stages of HIV (DiFranceisco et al. 2005; Weinhardt et al. 1999), such changes are especially important for newly infected individuals because of their heightened infectiousness (Cates et al. 1997; Koopman et al. 1997; Pilcher et al. 2004). In our data, partner reduction and serosorting were often driven by a desire to prevent transmitting the virus to other people. This altruistic motivation is encouraging, and offers a likely area of emphasis to sustain behavior change.

However, participants were much less successful at increasing condom use. They expressed pronounced discomfort and difficulties with condoms and, after diagnosis, the majority of reported sex acts remained unprotected. This finding stands in contrast to studies looking across risk groups, which observed relative increases in safer sex behaviors after diagnosis (DiFranceisco et al. 2005; Weinhardt et al. 1999). The absence of condom-related behavior change among our mostly MSM participants is likely due to serosorting, which our participants described specifically as an intended risk reduction strategy. This pattern of results is similar to those of Gorbach and colleagues (2006), who also observed increased serosorting but no increase in condom use among newly infected MSM, but is different than the findings of Colfax and colleagues (2002), who documented a decrease in unprotected anal intercourse but no shift toward serosorting. Clearly, consistent condom use is the ideal form of transmission-risk prevention. But, if a person is unable or unwilling to use condoms, serosorting offers a potential strategy for lowering the chances of HIV transmission. However, its success requires accurate assessments of partners' serostatuses (Butler and Smith 2007; Pinkerton 2008).

Although we do not know exactly how participants determined their partners' serostatuses, the accuracy of these assessments was almost certainly less than perfect. Only six participants reported engaging in risk behaviors with a person whom they knew to be HIV-positive in the months immediately prior to diagnosis, even though $100 \%$ (by definition) must have done so. And some participants noted explicitly that they learned after diagnosis that their ongoing sexual partners were in fact positive. Thus, it is likely that the pronounced post-diagnosis shift to serosorting was driven at least in part by changes in perception. That is, partners previously thought to be HIV-negative or of unknown serostatus were subsequently reclassified 
correctly as HIV-positive. This finding highlights the critical need to build skills to enhance communication and promote disclosure of sexual partners' HIV statuses.

A number of participants attributed aspects of their behavior change to loss of libido resulting from either seroconversion illnesses or the psychological adjustment to HIV infection. Both are temporary and undesirable states. In addition, seroconversion symptoms do not universally accompany new infections (Panel on Antiretroviral Guidelines for Adults and Adolescents 2008). Presumably, behavior changes prompted by these temporary conditions would dissipate as a person recovers, suggesting that interventions may need to focus attention on different motivators for longer-term behavior change. Nevertheless, it is important to note that even temporary reductions in risk behaviors during and immediately after AHI would help curtail the spread HIV within highly active sexual networks, given that the per-act probability of transmission is so high during this disease stage (Pilcher et al. 2004; Wawer et al. 2005).

Our findings come with a number of limitations. First, our sample consisted mainly of MSM recruited in urban environments. Thus, it is not clear to what degree the findings can be generalized. In particular, serosorting has been observed most frequently among gay men (Grov et al. 2007; Mao et al. 2006; Osmond et al. 2007; Parsons et al. 2005; Truong et al. 2006). It is possible that gay urban communities have unique social structures that facilitate serosorting (e.g., social events for HIV-positive men, norms about posting HIV status in online profiles, etc.). Second, our sample was small and comprised of individuals successfully diagnosed during acute/early infection. Most HIV infections are missed at these stages (Pilcher et al. 2005). Our participants may have had characteristics that made them more likely to be identified and more likely to reduce risk behaviors (e.g., unusually severe illnesses caused by seroconversion). Third, sexual risk data were obtained in structured surveys rather than via computerassisted methods. This mode of assessment may have resulted in less willingness to report socially undesirable behaviors (Gribble et al. 1999; Metzger et al. 2000; Turner et al. 1998). However, the structured survey was conducted after interviewers had established rapport with participants via the in-depth interviews, which may have helped mitigate this potential problem. Fourth, we did not receive from the clinical referral sites sufficient information to estimate the likely dates when participants were infected. As such, we cannot estimate how quickly after actual infection, as opposed to diagnosis, behavior changes occurred.

It is vital that we find ways to identify acute/early stage infections and to offer targeted interventions. Even temporary reductions in transmission risk behaviors during the first few months of infection could substantially reduce the spread of the disease within highly-active sexual networks (Cates et al. 1997; Koopman et al. 1997; Pilcher et al. 2005). This speaks to the need to raise awareness within high-risk population and sexual networks about the signs and symptoms of acute HIV infection, the necessity for regular and frequent testing among at-risk groups, the imperative to seek medical care/diagnosis, and the importance of refraining from high-risk sexual activities if the possibility of HIV infection is suspected (Stekler et al. 2006). The results of our study indicate that diagnosis with acute/early HIV infection is likely to promote some degree of short-term behavior change, but efforts will be needed to help people develop strategies for sustaining these changes.

Acknowledgments Primary funding for this study was provided by the National Institute of Mental Health as supplements to the following AIDS Research Centers: P30MH062246, Center for AIDS Prevention Studies, University of California San Francisco; P30MH043520, HIV Center for Clinical and Behavioral Research, New York State Psychiatric Institute and Columbia University; P30MH062512, HIV Neurobehavioral Research Center, University of California San Diego; P30MH052776, Center for AIDS Intervention Research, Medical College of Wisconsin; P30MH058107, Center for HIV Identification, Prevention and Treatment Services, University of California Los Angeles; and P30MH062294, Center for Interdisciplinary Research on AIDS, Yale University. Additional funding was provided by: P30AI42853, Lifespan/Tufts/Brown Center for AIDS Research and AI43638, Acute Infection and Early Disease Research Program, University of California San Diego. Complete details about funding, the study Steering Committee, co-investigators, collaborating scientists, and project staff are presented in the first paper of this series (Kerndt et al. 2009).

Open Access This article is distributed under the terms of the Creative Commons Attribution Noncommercial License which permits any noncommercial use, distribution, and reproduction in any medium, provided the original author(s) and source are credited.

\section{References}

Atkinson, J. H., Higgins, J., Vigil, O., Dubrow, R., Remien, R. H., Steward, W. T., et al. (2009). Psychiatric context of acute/early HIV infection. The NIMH Multisite Acute HIV Infection Study: IV. AIDS and Behavior. doi:10.1007/s10461-009-9585-3.

Butler, D. M., \& Smith, D. M. (2007). Serosorting can potentially increase HIV transmissions. AIDS (London, England), 21, 12181220. doi:10.1097/QAD.0b013e32814db7bf.

Cates, W. Jr., Chesney, M. A., \& Cohen, M. S. (1997). Primary HIV infection-A public health opportunity. American Journal of Public Health, 87, 1928-1930. doi:10.2105/AJPH.87.12.1928.

Colfax, G. N., Buchbinder, S. P., Cornelisse, P. G., Vittinghoff, E., Mayer, K., \& Celum, C. (2002). Sexual risk behaviors and implications for secondary HIV transmission during and after HIV seroconversion. AIDS (London, England), 16, 1529-1535. doi:10.1097/00002030-200207260-00010.

DiFranceisco, W., Pinkerton, S. D., Dyatlov, R. V., \& Swain, G. R. (2005). Evidence of a brief surge in safer sex practices after HIV testing among a sample of high-risk men and women. Journal of Acquired Immune Deficiency Syndromes, 39, 606-612.

Gorbach, P. M., Drumright, L. N., Daar, E. S., \& Little, S. J. (2006). Transmission behaviors of recently HIV-infected men who have 
sex with men. Journal of Acquired Immune Deficiency Syndromes, 42, 80-85. doi:10.1097/01.qai.0000196665.78497.f1.

Gribble, J. N., Miller, H. G., Rogers, S. M., \& Turner, C. F. (1999). Interview mode and measurement of sexual behaviors: Methodological issues. Journal of Sex Research, 36, 16-24.

Grov, C., DeBusk, J. A., Bimbi, D. S., Golub, S. A., Nanin, J. E., \& Parsons, J. T. (2007). Barebacking, the Internet, and harm reduction: An intercept survey with gay and bisexual men in Los Angeles and New York City. AIDS and Behavior, 11, 527-536. doi:10.1007/s10461-007-9234-7.

Kelly, J. A., Morin, S. F., Remien, R. H., Steward, W. T., Higgins, J. A., Seal, D. W., et al. (2009). Lessons learned about behavioral science and acute/early HIV Infection. The NIMH Multisite Acute HIV Infection Study: V. AIDS and Behavior. doi: 10.1007/s10461-009-9579-1.

Kerndt, P. R., Dubrow, R., Aynalem, G., Mayer, K. H., Beckwith, C., Remien, R. H., et al. (2009). Strategies used in the detection of acute/early HIV infections. The NIMH Multisite Acute HIV Infection Study: I. AIDS and Behavior. doi:10.1007/s10461009-9580-8.

Koopman, J. S., Jacquez, J. A., Welch, G. W., Simon, C. P., Foxman, B., Pollock, S. M., et al. (1997). The role of early HIV infection in the spread of HIV through populations. Journal of Acquired Immune Deficiency Syndromes and Human Retrovirology, 14, 249-258.

Mao, L., Crawford, J. M., Hospers, H. J., Prestage, G. P., Grulich, A. E., Kaldor, J. M., et al. (2006). 'Serosorting' in casual anal sex of HIV-negative gay men is noteworthy and is increasing in Sydney, Australia. AIDS (London, England), 20, 1204-1206. doi:10.1097/01.aids.0000226964.17966.75.

Metzger, D. S., Koblin, B., Turner, C., Navaline, H., Valenti, F., Holte, S., et al. (2000). Randomized controlled trial of audio computer-assisted self-interviewing: Utility and acceptability in longitudinal studies. HIVNET vaccine preparedness study protocol team. American Journal of Epidemiology, 152, 99-106. doi:10.1093/aje/152.2.99.

Osmond, D. H., Pollack, L. M., Paul, J. P., \& Catania, J. A. (2007). Changes in prevalence of HIV infection and sexual risk behavior in men who have sex with men in San Francisco: 1997-2002. American Journal of Public Health, 97, 1677-1683. doi: 10.2105/AJPH.2005.062851.

Panel on Antiretroviral Guidelines for Adults and Adolescents. (2008). Guidelines for the use of antiretroviral agents in HIV-1infected adults and adolescents. Department of Health and Human Services. Retrieved January 30, 2009, from http://www. aidsinfo.nih.gov/ContentFiles/AdultandAdolescentGL.pdf.

Parsons, J. T., Schrimshaw, E. W., Wolitski, R. J., Halkitis, P. N., Purcell, D. W., Hoff, C. C., et al. (2005). Sexual harm reduction practices of HIV-seropositive gay and bisexual men: Serosorting, strategic positioning, and withdrawal before ejaculation. AIDS, (London, England), 19(Suppl 1), S13-S25.

Pilcher, C. D., Fiscus, S. A., Nguyen, T. Q., Foust, E., Wolf, L., Williams, D., et al. (2005). Detection of acute infections during HIV testing in North Carolina. The New England Journal of Medicine, 352, 1873-1883. doi:10.1056/NEJMoa042291.

Pilcher, C. D., Tien, H. C., Eron, J. J. Jr., Vernazza, P. L., Leu, S. Y., Stewart, P. W., et al. (2004). Brief but efficient: Acute HIV infection and the sexual transmission of HIV. The Journal of Infectious Diseases, 189, 1785-1792. doi:10.1086/386333.

Pinkerton, S. D. (2008). Acute HIV infection increases the dangers of serosorting. American Journal of Preventive Medicine, 35, 184. doi:10.1016/j.amepre.2008.04.012.

Remien, R. H., Higgins, J. A., Correale, J., Bauermeister, J., Dubrow, R., Bradley, M., et al. (2009). Lack of understanding of acute HIV infection among newly-infected persons-Implications for prevention and public health. The NIMH Multisite Acute HIV Infection Study: II. AIDS and Behavior. doi:10.1007/s10461009-9581-7.

Stekler, J., Collier, A. C., Holmes, K. K., \& Golden, M. R. (2006). Primary HIV infection education: Knowledge and attitudes of HIV-negative men who have sex with men attending a public health sexually transmitted disease clinic. Journal of Acquired Immune Deficiency Syndromes, 42, 123-126. doi:10.1097/01.qai. 0000218357.82970.67.

Suarez, T. P., Kelly, J. A., Pinkerton, S. D., Stevenson, Y. L., Hayat, M., Smith, M. D., et al. (2001). Influence of a partner's HIV serostatus, use of highly active antiretroviral therapy, and viral load on perceptions of sexual risk behavior in a community sample of men who have sex with men. Journal of Acquired Immune Deficiency Syndromes, 28, 471-477.

Suarez, T., \& Miller, J. (2001). Negotiating risks in context: A perspective on unprotected anal intercourse and barebacking among men who have sex with men-Where do we go from here? Archives of Sexual Behavior, 30, 287-300. doi:10.1023/A:1002 700130455.

Truong, H. M., Kellogg, T., Klausner, J. D., Katz, M. H., Dilley, J., Knapper, K., et al. (2006). Increases in sexually transmitted infections and sexual risk behaviour without a concurrent increase in HIV incidence among men who have sex with men in San Francisco: A suggestion of HIV serosorting? Sexually Transmitted Infections, 82, 461-466. doi:10.1136/sti.2006.019 950.

Turner, C. F., Ku, L., Rogers, S. M., Lindberg, L. D., Pleck, J. H., \& Sonenstein, F. L. (1998). Adolescent sexual behavior, drug use, and violence: Increased reporting with computer survey technology. Science, 280, 867-873. doi:10.1126/science.280.5365. 867.

Van de Ven, P., Kippax, S., Crawford, J., Rawstorne, P., Prestage, G., Grulich, A., et al. (2002). In a minority of gay men, sexual risk practice indicates strategic positioning for perceived risk reduction rather than unbridled sex. AIDS Care, 14, 471-480. doi: 10.1080/09540120208629666.

Wawer, M. J., Gray, R. H., Sewankambo, N. K., Serwadda, D., Li, X., Laeyendecker, O., et al. (2005). Rates of HIV-1 transmission per coital act, by stage of HIV-1 infection, in Rakai, Uganda. The Journal of Infectious Diseases, 191, 1403-1409. doi:10.1086/ 429411.

Weinhardt, L. S., Carey, M. P., Johnson, B. T., \& Bickham, N. L. (1999). Effects of HIV counseling and testing on sexual risk behavior: A meta-analytic review of published research, 19851997. American Journal of Public Health, 89, 1397-1405. doi: 10.2105/AJPH.89.9.1397. 